\title{
FACET-II facility for advanced accelerator experimental tests
}

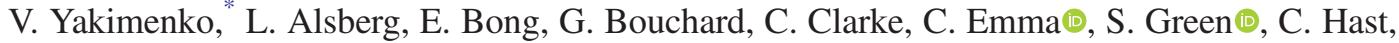 \\ M. J. Hogan, J. Seabury, N. Lipkowitz $\odot$, B. O’Shea, D. Storey, G. White, and G. Yocky® \\ SLAC National Accelerator Laboratory, Menlo Park, California 94075, USA
}

(Received 27 June 2019; published 9 October 2019)

\begin{abstract}
The National User Facility for Advanced Accelerator Experimental Tests II (FACET-II) at SLAC National Accelerator Laboratory expands upon the experiments conducted at FACET. Its purpose is to build upon the decades-long experience developed conducting accelerator R\&D at SLAC in the areas of advanced acceleration and coherent radiation techniques with high-energy electron and positron beams. This paper summarizes the motivations for the design and resulting capabilities of the FACET-II facility.
\end{abstract}

DOI: 10.1103/PhysRevAccelBeams.22.101301

\section{INTRODUCTION}

SLAC is a world leader in developing next-generation plasma wakefield acceleration (PWFA) technology for future accelerators (from compact systems to large-scale colliders) through operating the world's only high-energy electron and positron beam-driven plasma facility. The Facility for Advanced Accelerator Experimental Tests (FACET) was the centerpiece of the PWFA program, having operated as a Department of Energy (DOE) user facility from April 2012 until April 2016. FACET-II, the follow-on facility to FACET, is currently under construction. The DOE Advanced Accelerator Strategy Report defined a series of milestones for progress in advanced acceleration technology. These milestones define important areas of focus for the FACET-II facility, including preservation of beam quality and emittance, identifying techniques for positron acceleration in plasmas, developing plasma injectors as sources of ultralow-emittance beams, and demonstrating staging. FACET-II will provide beams optimized for the next generation of PWFA experiments and will be the only facility in the world capable of providing $10-\mathrm{GeV}$ electron and positron beams in support of accelerator science R\&D, with the primary focus on investigating key R\&D challenges of PWFA-based electron-positron colliders and fifth-generation light sources. The key research goals to be addressed and required accelerator hardware components to achieve these goals are summarized in Table I and further discussed below.

\footnotetext{
Corresponding author. yakimenk@slac.stanford.edu

Published by the American Physical Society under the terms of the Creative Commons Attribution 4.0 International license. Further distribution of this work must maintain attribution to the author(s) and the published article's title, journal citation, and DOI.
}

During the past two decades of research, the ultrarelativistic beam-driven PWFA concept has achieved many significant milestones. These include the demonstration of ultrahigh-gradient acceleration of electrons over meter-scale plasma accelerator structures [1], efficient acceleration of a narrow energy spread electron bunch at high gradients [2], positron acceleration using wakes in uniform plasmas [3] and in hollow plasma channels [4], the demonstration that highly nonlinear wakes in the "blow-out regime" have the electric field structure necessary for preserving the emittance of the accelerating bunch [5], tunable plasma-based energy dechirper [6], and acceleration of electrons in the plasma wakefield of a proton bunch [7].

The PWFA Collaboration has proposed an initial, fiveyear R\&D plan for FACET-II that is consistent with the DOE strategic plan [8]. A series of experiments have been proposed for FACET-II that are designed to demonstrate plasma wakefield acceleration where the drive beam is nearly depleted of its energy, the trailing bunch is accelerated with high efficiency while doubling its energy, and, ultimately, the emittance of the beam is preserved. Replacing the FACET thermionic injector and damping ring with a photoinjector in a configuration similar to that of the Linac Coherent Light Source (LCLS) [9] will enable FACET-II to investigate acceleration and beam quality preservation, utilizing beams with an emittance 2 orders of magnitude lower than what was routinely available at FACET. The resulting improvement in beam density will enable FACET-II to study new effects like the motion of plasma ions and unintended beam ionization in the plasma sources. Additional plasma-based experiments have been proposed for generating bunches with orders-of-magnitude lower emittance that will ultimately be needed both for an early application of a PWFA and for a plasma-based future linear collider [10]. For example, the development of ultrahigh-brightness beams may enable a new generation of high-gain free electron lasers that deliver fully coherent 
TABLE I. FACET-II summary of scientific motivations, performance goals, and design choices.

Research goal

High-Intensity driver to study PWFA relevant to linear colliders and user programs with intense electron beams

Positron PWFA and user programs with intense positron beams Acceleration of positrons in wake of electron drive beams

Studies of PWFA staging

Broad physics opportunities for interacting high-energy electrons, positrons, lasers, and plasmas
Accelerator component required

Photoinjector, 10-GeV linac, three-stage bunch compressor

Positron damping ring

Positron compression chicane

Compatibility with future witness injector installation

High-intensity laser, synchronization and interaction capabilities, specialized diagnostics hard x-ray pulses with terawatts of peak power [11]. With a potentially more compact footprint and enhanced capabilities, these systems could study electron dynamics in atoms and molecules within the resolution of the natural timescale of their motion. The new FACET-II photoinjector and upgraded bunch compressor systems have been designed to deliver high peak-current beams that allow plasma-based injector development at a relatively high plasma density, where the accelerating fields and output beam brightness are maximized. In addition to light source applications, these ultralow-emittance beams can serve as proxies for studying beam-plasma interactions with single bunches approaching linear collider intensities.

Novel simulations, diagnostics, and machine-learning techniques will be implemented to support delivery of higher-quality beams to enable the advanced acceleration programs and to simplify changing machine configurations for the needs of a variety of experiments. For example, FACET-II is expected to deliver beams with unprecedented properties (femtosecond duration and peak current $>200 \mathrm{kA}$ ) requiring new diagnostic approaches where the beam parameters cannot be measured directly. The application of machine-learning methods for predicting the longitudinal phase space distribution at FACET-II has been studied and tested. The approach consists of training a machine-learning-based virtual diagnostic to predict the longitudinal phase space using only nondestructive linac and $e$-beam measurements as inputs [12]. This approach has been validated with a simulation study for the FACETII linac and with an experimental demonstration conducted at LCLS - an important step towards showing the feasibility of implementing such a virtual diagnostic at FACET-II.

Most of the work to date has involved the acceleration of electrons, but positrons are also required for future colliders. Accelerating a trailing positron bunch in a plasma is more challenging, as the plasma response can be asymmetric for positrons and electrons. Energy gain by a distinct trailing positron bunch in a plasma wakefield accelerator was demonstrated at FACET, spanning nonlinear to quasilinear regimes, and unveiled the beam loading process underlying the accelerator energy efficiency. A positron bunch was used to drive the plasma wake in the experiment, though the quasilinear wake structure could as easily be formed by an electron bunch or a laser driver. The results marked the first acceleration of a distinct positron bunch in plasma-based particle accelerators [13]. Hollow channel plasma wakefield acceleration is a proposed method to provide high-acceleration gradients for electrons and positrons alike: a key to future lepton colliders. However, beams which are misaligned from the channel axis induce strong transverse wakefields, deflecting beams and reducing collider luminosity. This undesirable consequence sets a tight constraint on the alignment accuracy of the beam propagating through the channel. Direct measurements of beam misalignment-induced transverse wakefields are therefore essential for designing mitigation strategies [14]. Phased upgrades to FACET-II are expected to provide stable production of high peak-current positrons with nanoCoulomb charge using the existing FACET positron source and FACET-II $10 \mathrm{GeV}$ linac. This will represent a capability unique in the world, enabling experimental investigations into the optimal technique for high-gradient positron acceleration in plasma. The addition of a second beam line in the final bunch compressor (in sector 20) will allow simultaneous delivery of electron and positron bunches to the plasma and, for the first time, allow linear collider relevant studies of positron acceleration on the wake of an electron bunch.

The combination of high-intensity electron and positron beams, a synchronized high-power laser, and interaction regions with specialized diagnostics opens the door to an even broader experimental program including dielectric wakefield acceleration [15], diverse physics experiments such as strong-field QED, and testing technology needed to develop demonstration facilities. One example of testing technology at FACET-II for future demonstration facilities is a series of experiments to characterize filamentationbased gamma-ray sources. When an electron beam propagates through a plasma, the resulting plasma electron return currents are established as a reaction to the beam. The counterstreaming beam and plasma electrons result in a current filamentation instability [16] and form selfgenerated beam filaments and electromagnetic fields. Trajectories of the beam electrons are bent in this beaminduced, and instability-enhanced, magnetic field, and synchrotron radiation is emitted. Theoretically consistent scaling of transverse filament size was observed over a wide range of plasma densities in experiments at BNL's Accelerator Test Facility using 60-MeV beams [17]. Recent 
theoretical and numerical work has shown that a significant quantity of the electron beam energy, potentially exceeding $10 \%$, can be converted into gamma rays using high-energy electron beams in a high-density plasma [18].

Designing the capability to produce record beam currents at FACET-II (potentially as high as $200 \mathrm{kA}$ ) was motivated by the extreme beam parameters required to conduct experimental research on relativistic plasma instabilities and gamma-ray sources of unprecedented efficiency and brightness. The timeline for FACET-II has nominal electron beam parameters $(10 \mathrm{GeV}, 2 \mathrm{nC}, 15 \mathrm{kA}$, and $30 \mathrm{~Hz}$ ) available in 2020 and nominal positron parameters $(10 \mathrm{GeV}, 1 \mathrm{nC}, 6 \mathrm{kA}$, and $5 \mathrm{~Hz})$ in 2022. FACET-II is expected to operate 6 months per year. Experimental scheduling will balance the increased reliability and flexibility of the photoinjector against less-frequent tunnel access when LCLS-II is in operation.

Below, we describe the expected performance of the accelerator, including beam parameters for the present set of operational modes, as well as the performance of the experimental laser and diagnostics.

\section{ACCELERATOR OVERVIEW}

The FACET-II accelerator design [19] consists of a $135-\mathrm{MeV}$, high-brightness photoinjector constructed in an off-axis injection line in sector 10 of the SLAC linac, two four-bend chicane bunch compressors, with a third and final compression stage provided in sector 20. The existing FACET sector 20 bunch compressor was redesigned for maximum peak-current throughput and minimal emittance degradation via coherent synchrotron radiation (CSR) and is capable of generating peak bunch currents in excess of $200 \mathrm{kA}$, at beam energies up to $13 \mathrm{GeV}$. The rf gun is capable of generating $3 \mu \mathrm{m}$-rad emittance electron pulses at $2 \mathrm{nC}$ bunch charge. The deliverable beam quality into sector 20 depends upon the desired bunch compression and varies between high-quality $\sim 0.1 \%$ energy spread, $3 \mu \mathrm{m}-\mathrm{rad}$ emittance beams at low compression to $\sim 1 \%$ energy spread, higher-emittance beams at full compression. Positron operations are achieved through the reuse of the existing positron target and the introduction of a new, ultracompact 335-MeV damping ring [20]. Simulation studies show sufficient quality positron beams of at least $1 \mathrm{nC}$ can be extracted from the damping ring and injected into the main linac in sector 11 . The possibility for simultaneous delivery of both electrons and positrons can be achieved through a further upgrade that adds positron path sections to the bunch compressors BC14 and BC20. The FACET-II accelerator complex is to be installed in the central $1 \mathrm{~km}$ of the main SLAC linac, between sectors 10 and 20 as depicted in the schematic in Fig. 1.

The injector, three bunch compressor stages, and the final focus system are designed to allow flexibility in beam parameters delivered to the experimental area. The electron bunch length can be varied from an initial $\sim 1 \mathrm{~mm}$ exiting the injector down to submicrometer lengths in sector 20. The bunch charge from the rf photoinjector can be varied up to $2 \mathrm{nC}$ and in the future possibly higher (up to $\sim 5 \mathrm{nC}$ ). Positron bunches with at least $1 \mathrm{nC}$ of charge can be generated by the positron production system (driven by 4-nC electron bunch drive pulses) and cooled in a damping ring. Delivered emittances of electron and position beams depend nontrivially on the choice of bunch charge and degree of longitudinal compression.

To meet the needs of the proposed experiments, other FACET-II machine parameters are configured in three categories with the associated beam parameters: twobunch, single-bunch high density, and single-bunch high quality. All parameters are quoted at the sector 20 experimental interaction region. Machine parameters common to all operation modes are shown in Table II for electron and positron beam options.

\section{A. Two-bunch mode (electrons)}

To serve the PWFA accelerator R\&D program, we propose generating a double laser pulse on the rf photocathode (separated by $\sim 7 \mathrm{ps}$ ), coaccelerating this double pulse through the linac, and arranging for high peak-current drive and witness pulses at the sector 20 interaction region with 500 fs temporal spacing (see Table III).

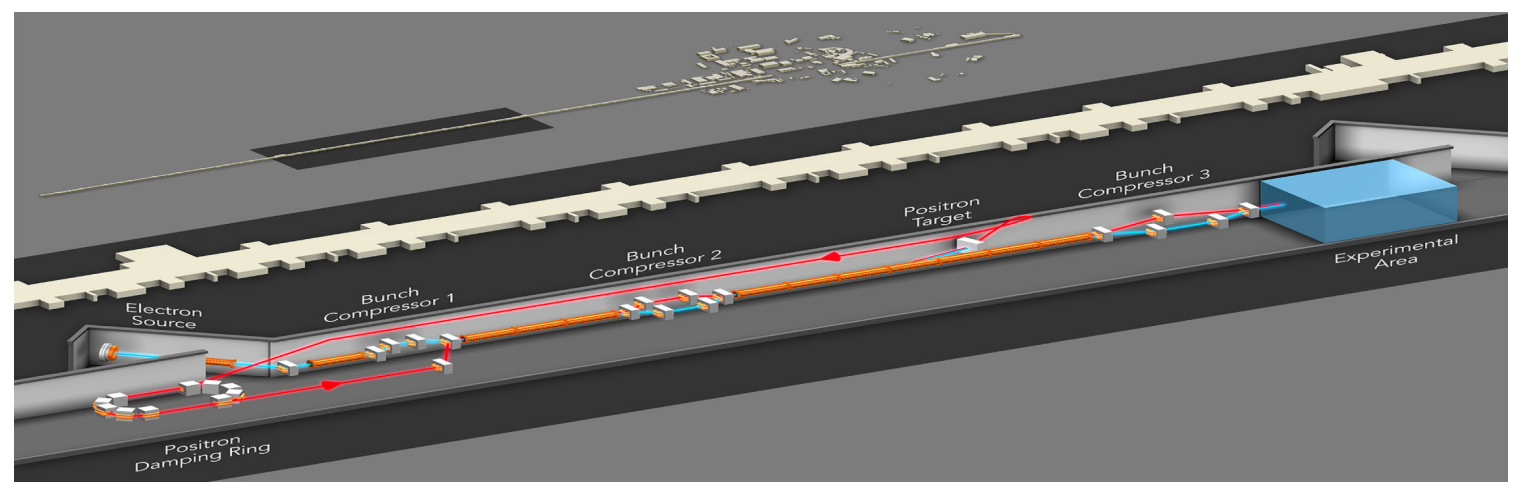

FIG. 1. Schematic layout of FACET-II in the middle third of the SLAC linac, downstream of LCLS-II, and upstream of LCLS-I. 
TABLE II. Common machine parameters for all following FACET-II configurations.

\begin{tabular}{lcc}
\hline \hline Parameter & $e^{-}$ & $e^{+}$ \\
\hline Injected energy @ sector $11, E_{i}, \mathrm{MeV}$ & \multicolumn{2}{c}{335} \\
Pulse rep. rate $f_{\text {rep }}, \mathrm{Hz}$ & $1-30$ & $1-5$ \\
Emittance into sector $20, \gamma \varepsilon_{x, y}, \mu$ m-rad & $3-4$ & 13 \\
\hline \hline
\end{tabular}

TABLE III. Two-bunch mode parameters. $\beta^{*}$ refers to the optical beta function magnitude at the interaction waist in sector 20

\begin{tabular}{lcc}
\hline \hline Parameter (units) & Drive pulse & Witness pulse \\
\hline Final beam energy $E_{f}(\mathrm{GeV})$ & \multicolumn{2}{c}{10.0} \\
Bunch charge $Q_{b}(\mathrm{nC})$ & 1.3 & 0.6 \\
Peak current $I_{p k}(\mathrm{kA})$ & 30 & 15 \\
$\beta^{*}(\mathrm{~cm})$ & $5-50$ & \\
Bunch spacing $(\mu \mathrm{m})$ & 150 & \\
Final rms energy spread, dE/E $(\%)$ & 0.8 & 0.3 \\
\hline \hline
\end{tabular}

\section{B. Single-bunch, high charge density mode (electron)}

Filamentation experiments, future nonlinear QED experiments, and others benefit from having as high peak-current and/or high 3D charge density beams as possible delivered to the sector 20 interaction point (see Table IV).

\section{Single-bunch, high-quality mode (electron)}

For initial strong-field QED experimental studies to characterize the FACET laser-beam interaction, a stable beam with well-defined Gaussian profile characteristics is desired. This mode of operation is also desirable for the initial commissioning of the accelerator. This is achieved by using moderate compression and focusing settings while operating at as high an energy as stably achievable
TABLE IV. Single-bunch, high Q mode parameters.

\begin{tabular}{lc}
\hline \hline Parameter (units) & Value \\
\hline Final beam energy $E_{f}(\mathrm{GeV})$ & 10.0 \\
Bunch charge $Q_{b}(\mathrm{nC})$ & 2.0 \\
Peak current $I_{p k}(\mathrm{kA})$ & $50-200$ \\
$\beta^{*}(\mathrm{~cm})$ & $5-100$ \\
Final rms energy spread, dE/E $(\%)$ & 1.2 \\
\hline \hline
\end{tabular}

TABLE V. Single-bunch, high-quality mode parameters.

\begin{tabular}{lc}
\hline \hline Parameter (units) & Value \\
\hline Final beam energy $E_{f}(\mathrm{GeV})$ & 13.0 \\
Bunch charge $Q_{b}(\mathrm{nC})$ & 2.0 \\
rms bunch length $\sigma_{z}(\mathrm{~mm})$ & 0.1 \\
$\beta^{*}(\mathrm{~m})$ & 10 \\
Final rms energy spread, dE/E $(\%)$ & 0.05 \\
\hline \hline
\end{tabular}

to minimize coherent effects in the $\mathrm{BC} 20$ compressor chicane. A final beam energy of $13 \mathrm{GeV}$ is possible by operating all L3 accelerating structures on crest while still maintaining the designated longitudinal feedback and spare stations to maintain tunability (see Table V).

\section{Kraken waist configuration (electrons)}

In addition to the three experimental area configurations outlined above, an upstream waist location is provided in the final focus system in sector 20 for additional experiments within the so-called "Kraken chamber." The Kraken chamber is a device installed to accommodate various experimental installations in an upstream area of sector 20 (as can be seen in Fig. 2). Two configurations are supported for this location: with either round or flat beams at the waist (flat means a 100:1 ratio of the beta function at the waist). See Table VI.

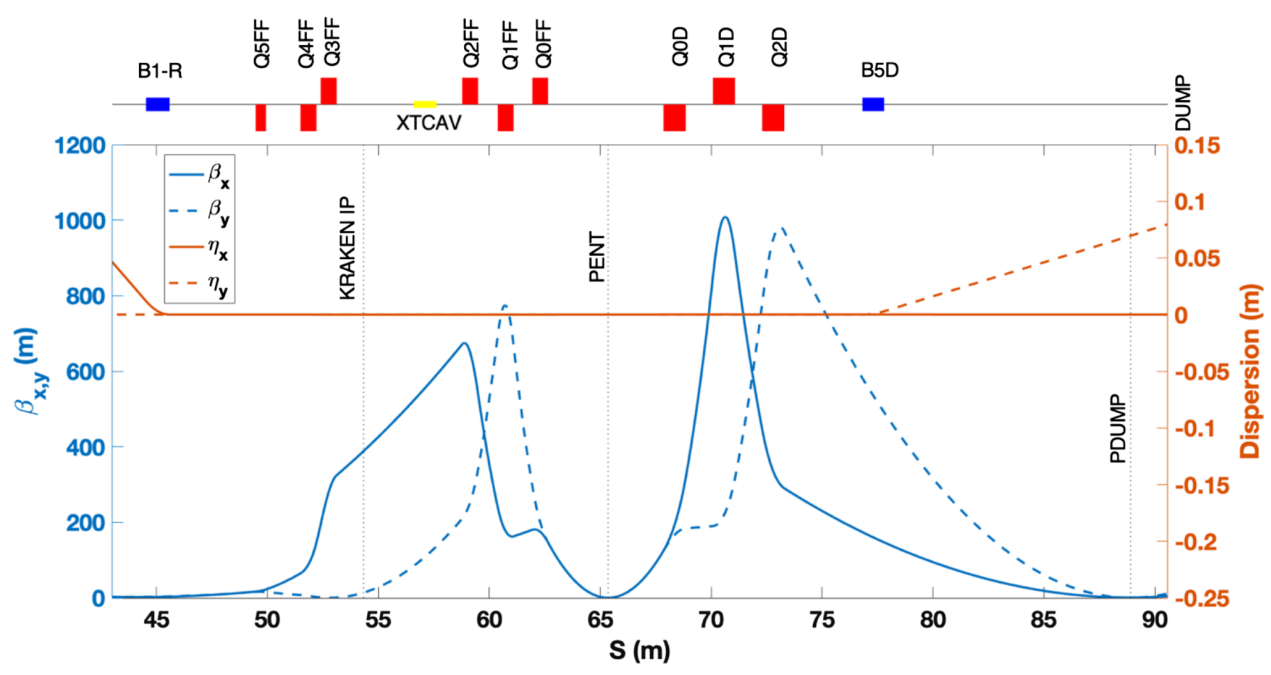

FIG. 2. Beam optical functions in the interaction region with focusing set for PWFA experiments and an imaging condition in the electron beam energy spectrometer. 
TABLE VI. Upstream (Kraken) waist parameters.

\begin{tabular}{|c|c|c|}
\hline Parameter (units) & Round waist & Flat waist \\
\hline Final beam energy $E_{f}(\mathrm{GeV})$ & \multicolumn{2}{|c|}{10.0} \\
\hline Bunch charge $Q_{b}(\mathrm{nC})$ & \multicolumn{2}{|c|}{2.0} \\
\hline Peak current $I_{p k}(\mathrm{kA})$ & \multicolumn{2}{|c|}{$3-50$} \\
\hline$\beta^{*}(\mathrm{~m})$ (horizontal) & 0.2 & 0.15 \\
\hline$\beta^{*}(\mathrm{~m})$ (vertical) & 0.2 & 15 \\
\hline Final rms energy spread, dE/E (\%) & \multicolumn{2}{|c|}{1.2} \\
\hline
\end{tabular}

TABLE VII. Single-bunch, positron mode parameters.

\begin{tabular}{lc}
\hline \hline Parameter (units) & Value \\
\hline Final beam energy $E_{f}(\mathrm{GeV})$ & 10.0 \\
Bunch charge $Q_{b}(\mathrm{nC})$ & 1.0 \\
Final peak current $I_{p k}(\mathrm{kA})$ & 6 \\
$\beta^{*}(\mathrm{~cm})$ & $5-100$ \\
Final rms energy spread, dE/E $(\%)$ & 0.7 \\
\hline \hline
\end{tabular}

TABLE VIII. Simultaneous electron + positron mode parameters.

\begin{tabular}{lcc}
\hline \hline Parameter (units) & $e^{-}$ & $e^{+}$ \\
\hline Final beam energy $E_{f}(\mathrm{GeV})$ & 10.0 & \\
Bunch charge $Q_{b}(\mathrm{nC})$ & 2.0 & 1.0 \\
Final peak current $I_{p k}(\mathrm{kA})$ & 30 & 6 \\
$\beta^{*}(\mathrm{~cm})$ & $5-100$ & \\
Final rms energy spread, dE/E $(\%)$ & 1.2 & 0.7 \\
\hline \hline
\end{tabular}

\section{E. Single positron bunch mode}

A 1-nC bunch of positrons is to be extracted at up to $5 \mathrm{~Hz}$ from a damping ring in sector 10 and injected into the main linac in sector 11, driven by a 4-nC electron bunch striking the positron target in sector 19. This design is to deliver a single bunch with the maximum possible peak current to the sector 20 experimental region to be used for the PWFA experimental program (see Table VII).

\section{F. Simultaneous electron + positron operation mode}

Upon construction of the positron systems and positron arm of the $\mathrm{BC} 20$ chicane, coacceleration of electrons and positrons (with half-bucket spacing in the main s-band $\mathrm{rf}$ linac) is possible. The two bunches are to be brought into simultaneous longitudinal positions by passing through the positron arm of BC20 with $\pm 500 \mu \mathrm{m}$ static and $\pm 100 \mu \mathrm{m}$ dynamic adjustment capabilities for the relative $z$ positions at the interaction region. Dynamic variability is achieved using a pair of adjustable minichicanes within the main positron arm of BC20 (see Table VIII).

\section{G. Beam optics for the experimental region}

After the final bunch compressor in sector 20 of the SLAC linac, a final focus system consisting of two quadrupole triplets is used to control the beam properties in the experimental region. Figure 2 shows the magnet configuration required to deliver beam to the PWFA experiments (interaction point labeled as "PENT") with the smallest beam size ( $5 \mathrm{~cm}$ beta function). A third quadrupole triplet after the main experimental region refocuses the beam to a downstream experimental station immediately upstream of the main beam dump (labeled "PDUMP"). A vertical bend magnet directs the beam downwards toward the dump and provides vertical dispersion for energy spectrometry. An additional interaction region can be provided after the first triplet (labeled "KRAKEN IP"): Figure 3 shows such a configuration with a round beam at the KRAKEN IP. Additionally, a configuration with a 100:1 beta function ratio at the KRAKEN IP is possible. Finally, a transverse deflecting cavity is located within the final focus system as

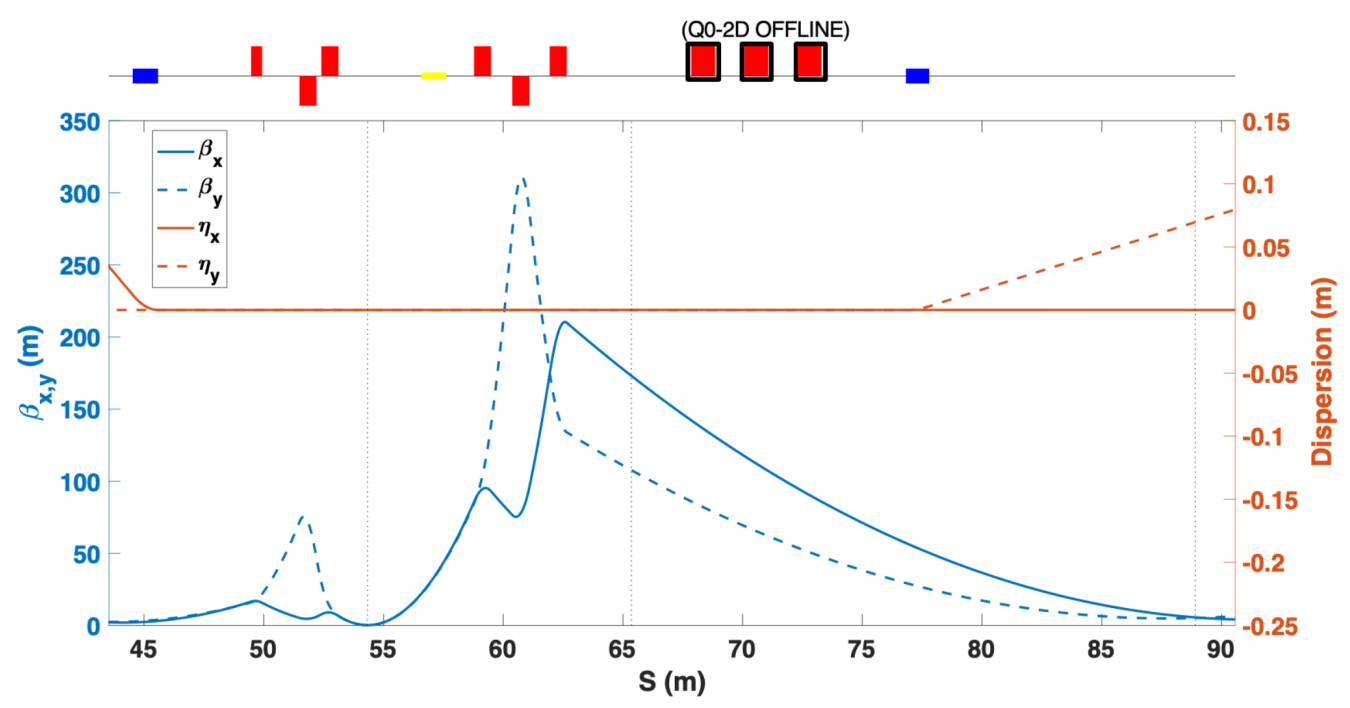

FIG. 3. Beam optical functions in the interaction region with focusing set for the alternative, upstream IP location. 


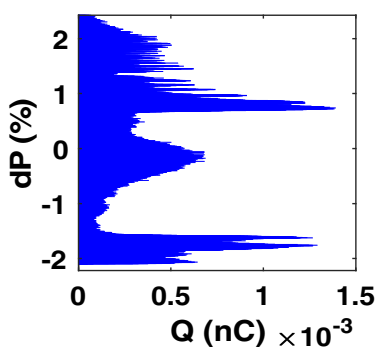

Mean Energy $=10.000 \mathrm{GeV}$
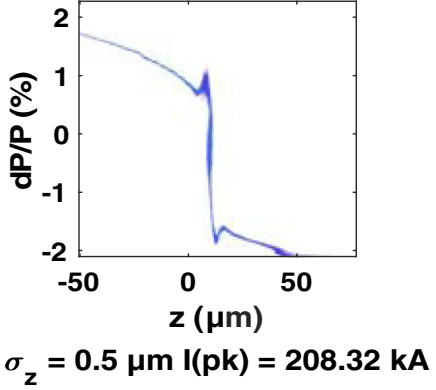

rms dP/P $=1.24508 \%$

rms $Z=32.8883 \mu \mathrm{m}$

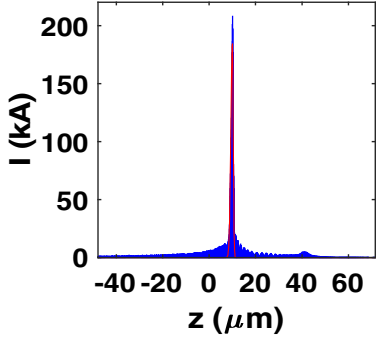

FIG. 4. Longitudinal phase space of the 2-nC electron bunch tracked to sector 20 at maximum compression.
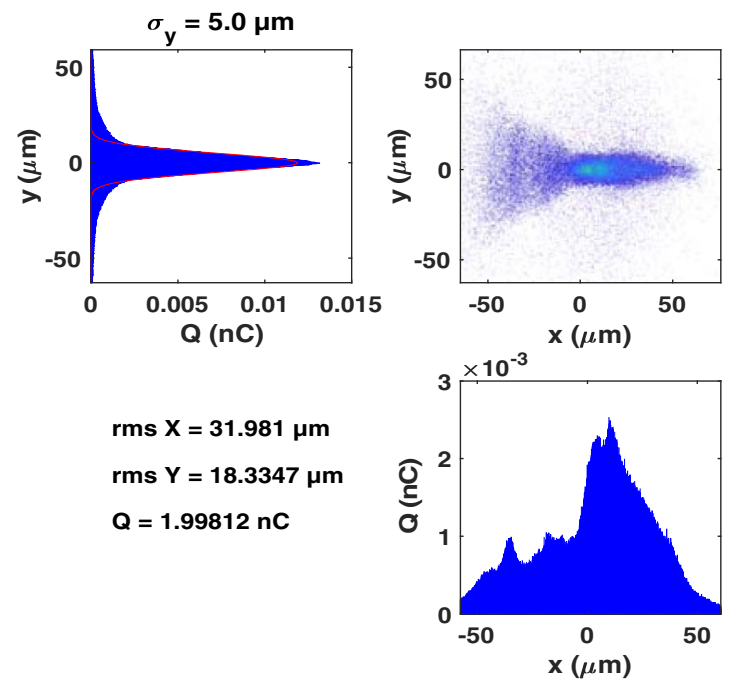

FIG. 5. Transverse projections of the 2-nC electron bunch tracked to sector 20 at maximum compression.

depicted in these figures (labeled "XTCAV"). It streaks the beam horizontally, allowing for reconstruction of the longitudinal phase space (energy-bunch length profile) at PDUMP.

\section{H. Start-to-end tracking}

Particles for positrons and electrons are tracked from their sources through to the experimental area. The tracking code GPT [21] is used for the electron injector, BMAD [22] is used for the positron damping ring, and Lucretia [23] is used for all other beam transportation.

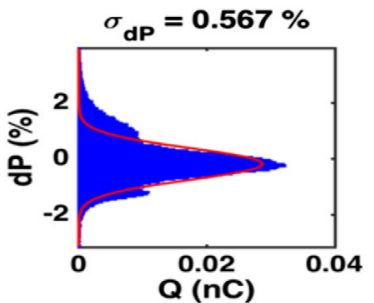

Mean Energy $=10.000 \mathrm{GeV}$
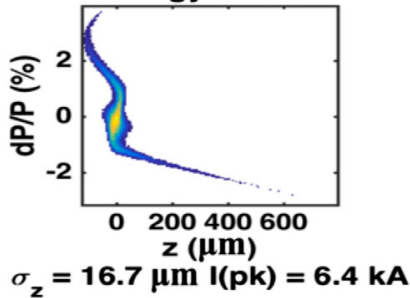

rms dP/P $=0.832536 \%$ rms $Z=37.4579 \mu \mathrm{m}$

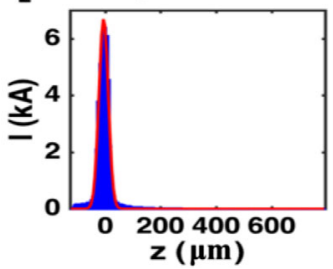

FIG. 6. Longitudinal phase space of the 2-nC bunch tracked to sector 20 at limited to $6 \mathrm{kA}$ peak current compression.
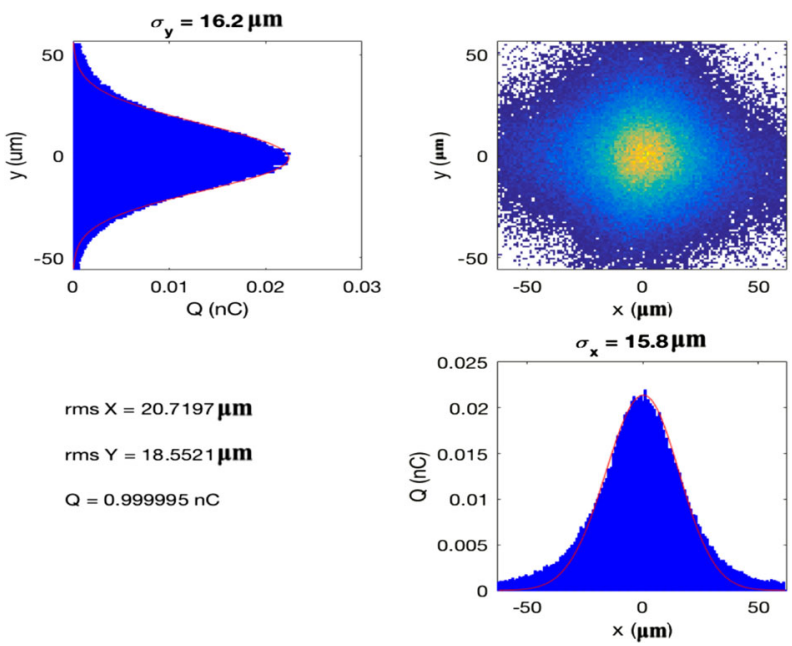

FIG. 7. Transverse projections of the 1-nC positron bunch tracked to sector 20 with $6 \mathrm{kA}$ peak current compression.

The tracking of electron bunches includes 3D spacecharge effects in the injector as well as longitudinal space-charge effects in the linac. Incoherent and coherent synchrotron radiation effects are included for the bends of the bunch compressors, and longitudinal and transverse wakefield effects are included for the linac structures. The positron-tracking model includes intrabeam scattering effects in the damping ring, which is operated in a fully coupled mode in order to extract a round beam for injection into the linac.

Figures 4 and 5 show a maximally compressed 2-nC electron bunch tracked to the sector 20 interaction region, displaying significant nonlinear aberrations caused by microbunching instabilities and CSR, mainly in the final compressor chicane. The CSR effects in the final compressor bend are strongly pronounced due to the very short 

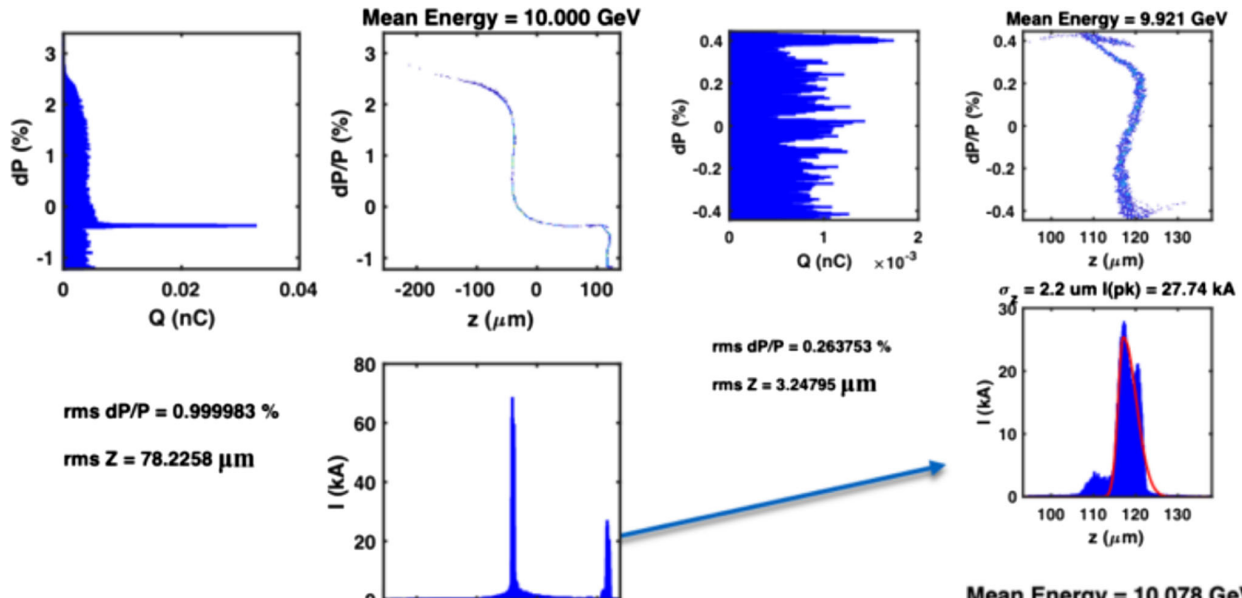

$\Delta \mathrm{z}=150 \mu \mathrm{m}$

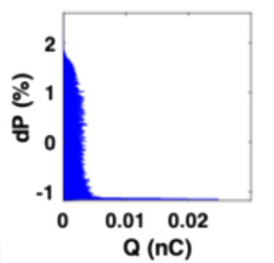

Mean Energy $=10.078 \mathrm{GeV}$

$(\Delta \mathrm{t}=500 \mathrm{fs})$ (peak-peak)
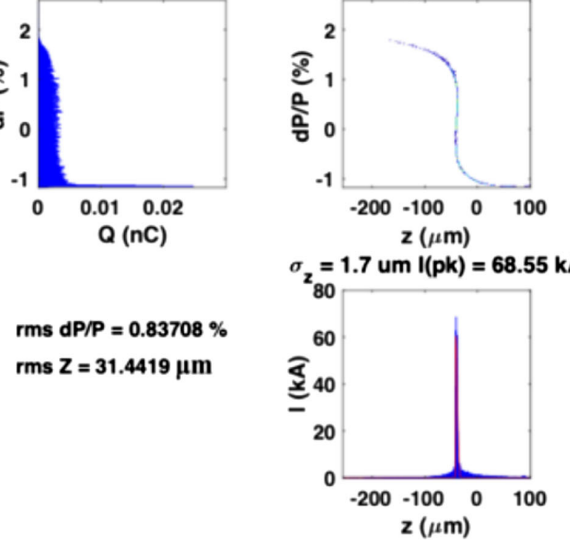

FIG. 8. Longitudinal phase space at sector 20 interaction point for the two-bunch configuration. From the tracking simulation.
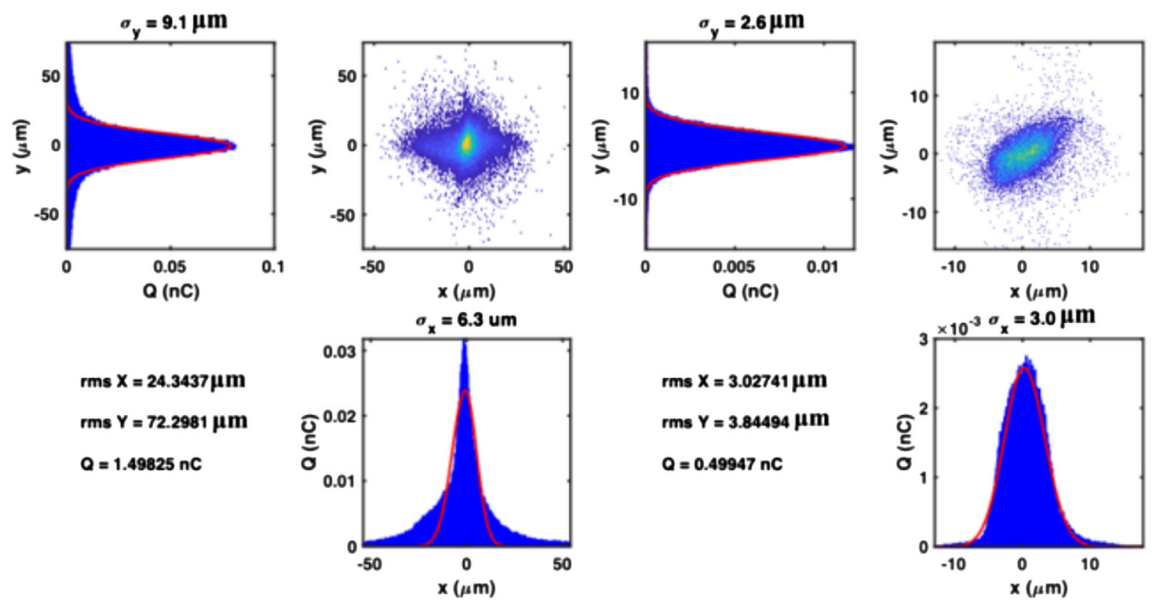

\section{Drive Bunch}

\section{Witness Bunch}

$$
\begin{array}{l|l}
\gamma \varepsilon_{x, y}=35.0,115 \mu \mathrm{m}-\mathrm{rad} & \gamma \varepsilon_{x, y}=3.7,4.1 \mu \mathrm{m} \text {-rad } \\
\gamma \varepsilon_{x, y}(90 \%)=20.6,37.1 \mu \mathrm{m}-\mathrm{rad} & \gamma \varepsilon_{x, y}(90 \%)=3.2,2.6 \mu \mathrm{m} \text {-rad }
\end{array}
$$

FIG. 9. Tracked transverse two-bunch mode particle distributions at sector 20 interaction point for the leading "drive" bunch and trailing "witness" bunch. 
bunch length and are the subject of ongoing research as to how to correctly model this effect and possible ways to mitigate the ensuing beam degradation. The final emittance in this case is $68 \times 6.6 \mathrm{~mm}-\mathrm{mrad}$. In contrast, the strongfield QED (SFQED) configuration generates relatively pure Gaussian transverse distributions with $<4 \mathrm{~mm}-$ mrad final emittances.

Figures 6 and 7 show the longitudinal and transverse dimensions of the tracked positron particles at the sector 20 interaction region waist. The peak current for the positron pulse is limited to about $6 \mathrm{kA}$ due to the necessarily larger initial longitudinal emittance of the beam in the damping ring.

The results of start-to-end tracking simulations for the two-bunch configuration are shown in Figs. 8 and 9 below. The double-pulsed charge profile is simulated in the GPT injector simulation and tracked through the linac and various bunch compressors. Both "drive" and "witness" bunches are transported in the same s-band rf bucket. Because of the increased longitudinal extent of this beam structure compared with the single-bunch case above, considerable nonlinear distortions are seen. There is a $150-\mathrm{MeV}$ energy differential between drive and witness bunches in sector 20. The optics have been tuned to preserve the beam quality of the witness bunch at the expense of degrading the drive bunch quality.

\section{Simulations of particle transport stability}

A Monte Carlo simulation was constructed to assess the pulse-to-pulse variation in the key deliverable performance parameters (emittance at the end of the linac, bunch length, and peak current at the sector 20 IP). Using a 100-seed

TABLE IX. rms jitter parameters used in the Monte Carlo tracking simulation.

\begin{tabular}{lc}
\hline \hline Property & Value \\
\hline Source charge fluctuation & $1 \%(e-), 2 \%(e+)$ \\
Source position fluctuation & $0.05,0.2 \sigma(e-, e+)$ \\
Initial electron laser timing jitter & $200 \mathrm{fs}$ \\
L1X phase jitter & $0.25 \mathrm{degX}$ \\
L1S phase jitter & $0.1 \mathrm{degS}$ \\
L2 phase jitter & $0.25 \mathrm{degS}$ \\
L3 phase jitter & $0.25 \mathrm{degS}$ \\
L0P phase jitter & $0.1 \mathrm{degS}$ \\
L1X amplitude jitter & $0.25 \%$ \\
L1S amplitude jitter & $0.1 \%$ \\
L2 amplitude jitter & $0.25 \%$ \\
L3 amplitude jitter & $0.25 \%$ \\
L0P amplitude jitter & $0.25 \%$ \\
BC0 magnet strength jitter & $10^{-5} \mathrm{~dB} / \mathrm{B}$ \\
BC1 magnet strength jitter & $10^{-4} \mathrm{~dB} / \mathrm{B}$ \\
BC2 magnet strength jitter & $10^{-4} \mathrm{~dB} / \mathrm{B}$ \\
BC3 magnet strength jitter & $10^{-4} \mathrm{~dB} / \mathrm{B}$ \\
Magnet vibration (x/y) & $1.5 / 0.5 \mu \mathrm{m}$ \\
\hline \hline
\end{tabular}

TABLE X. Results from the Monte Carlo tracking simulation study using jitter data from above table. Design requirements and results from 100 seed simulation runs are shown. The simulation results show the mean and rms spread of results obtained.

\begin{tabular}{lcccr}
\hline \hline Parameter & $\begin{array}{c}\text { Electron } \\
\text { bunch Des. }\end{array}$ & $\begin{array}{c}\text { Positron } \\
\text { bunch Sim. }\end{array}$ & Des. & Sim. \\
\hline$\varepsilon_{x, n}(\mu \mathrm{m}-\mathrm{rad})$ & $<20$ & $4.4 \pm 0.5$ & $<20$ & $10.7 \pm 0.7$ \\
$\varepsilon_{y, n}(\mu \mathrm{m}-\mathrm{rad})$ & $<20$ & $3.3 \pm 0.1$ & $<20$ & $13.0 \pm 0.2$ \\
$\sigma_{z}(\mu \mathrm{m})$ & $<20$ & $3.1 \pm 1.5$ & $<20$ & $16.5 \pm 0.2$ \\
$I_{p k}(\mathrm{kA})$ & $>10$ & $64 \pm 16$ & $>5$ & $5.8 \pm 0.2$ \\
\hline \hline
\end{tabular}

simulation with expected pulse-to-pulse error parameters (as shown in Table IX), the mean and rms fluctuations calculated are shown in Table $\mathrm{X}$ for the single-bunch electron and positron cases (where the electron beam is configured to provide a more conservative 65 -kA peak current compared with the case shown above). The measured arrival time jitter for both electron and positron pulses was $100 \mathrm{fs}$ rms.

\section{EXPERIMENTAL AREA}

The FACET-II experimental area is designed to accommodate a range of simultaneous experiments. Emittance preservation in PWFAs requires beam qualities that are possible only by using a differential pumping system. The differential pumping system is designed to enable 5 Torr helium operation in the experimental area suitable for the full suite of plasma-based experiments discussed above. To coalign the electron beam and experimental laser, the experimental area includes optical transition radiation (OTR) and yttrium aluminum garnet (YAG) beam diagnostics with typical resolutions of $15 \mu \mathrm{m}$. Temporal overlap is measured using an electro-optic sampling beam-laser timing diagnostic [24] with a resolution of $50 \mathrm{fs}$, necessary for Trojan horse [11] and plasma wakefield imaging. Electron injection and ionization laser optics are housed in a vacuum chamber upstream of a meter-long plasma section of beam line. In-vacuum motors with micrometer precision are used to position the laser optics as well as electron injection and filamentation targets. The meter-long plasma section of the experimental area can be remotely shifted on and off the beam axis as needed for PWFA emittance preservation, transverse wakefield experiments, and staging using injected electron beams.

Plasma sources vary depending on the specific goals of each experiment and are designed by the individual experimental collaborations. The anticipated experimental program currently requires support for three types of plasma sources: beam and laser ionized meter-scale alkali-metal heat pipe ovens [25], meter-scale laser ionized static fill gas sections, and 1-5-cm-long pulsed gas jets. The meter-long plasma section currently supports the use of lithium, rubidium, helium, hydrogen, argon, and nitrogen for generating plasma. To support these various plasma sources, the experimental area has been designed to provide 
the required physical space and the required gas delivery and vacuum systems including differential pumping systems as well as an experimental laser system that can be used for preionization of the plasma medium of choice.

\section{A. Laser}

The FACET-II experimental laser is a 20-TW Ti: sapphire-based system that is designed to meet the diverse requirements of the FACET-II experimental program. The laser provides the coherent light necessary for plasma ionization, electron injection, plasma wakefield imaging, and SFQED experiments. The laser uses a Vitara oscillator, a Legend Elite regenerative amplifier, and two stages of multipass amplification. The Vitara oscillator cavity is actively locked to the rf signal, which sets the timing for the accelerator. Based on the experience at FACET and LCLS, the time of arrival jitter between electron and laser beams is expected to be less than $50 \mathrm{fs}$ (rms). Bandwidthlimited 35-fs pulses necessary for precise, local ionization experiments such as the Trojan horse, are achieved by using a Dazzler and Wizzler [26]. The laser has a flattop transverse profile with phase errors of less than $50 \mathrm{~nm} \mathrm{rms}$, which enables the high intensities required for SFQED experiments, provided by an Imagine Optics deformable mirror. Near-field and far-field cameras, in addition to wavefront sensors, are used to monitor the mode quality on a shot-by-shot basis. For Trojan horse and wakefield imaging experiments, part of the laser is split off in the tunnel and separately transported to interaction points along the beam line. This "probe" laser is separately compressed and adjustable by \pm 250 ps with respect to the main laser. Both the main and probe laser use in-house active pointing stabilization to increase the time the laser is delivered to users. The FACET-II experimental laser is designed to accommodate two upgrade paths with minimal laser modification. The addition of a GAIA or GAIA-HP (or similar) pump laser and larger compressor gratings enables 40- and 100-TW operation, respectively.

\section{B. Diagnostics}

The reimaging spectrometer downstream of the interaction region provides diagnostics for electrons and gamma radiation (see Figure 11). The projected beam emittance is quantified using the butterfly technique [27]. This uses a magnet triplet and dipole in conjunction with a diffractionlimited imaging system with a resolution of $4 \mu \mathrm{m}$. The system is designed to measure $2-100 \mu \mathrm{m}$ emittances with $5 \%$ accuracy, which is necessary for PWFA emittance preservation and transverse wakefield studies. To reach 5\% accuracy at the lowest emittances, the electron beam and light collection optics have been optimized for high resolution [28]. The required rms energy spread is $\mathrm{dE} / E=0.2 \%$. Additionally, a pair of phosphor and Cherenkov light profile monitors provide $0.4 \%$ energy resolution to measure pump depletion of the drive beam in PWFA experiments [29].
A set of phosphor screens sensitive to $30 \mathrm{keV}$ to $100 \mathrm{MeV}$ gamma radiation emitted in the experimental area will determine angular and spectral information to be used to probe the electron beam dynamics within plasmas and SFQED experiments and emittance growth in PWFA experiments. Compton and bent crystal-based spectrometers are being developed to provide resolved double-differential angles and energy spectra of incoming photons in the range of $\mathrm{keV}$ to $30 \mathrm{MeV}$ [30]. To characterize the high-energy gamma-radiation spectra emitted by SFQED and filamentation experiments, a pair spectrometer will be installed in the beam transport prior to the electron beam dump, sensitive to photons in the range of $\mathrm{MeV}$ to $10 \mathrm{GeV}$.

A 20-MV X-band deflecting cavity providing a horizontal deflection will be used in conjunction with the imaging spectrometer to deliver a single-shot measurement of the longitudinal phase space with $2 \mu \mathrm{m}$ (6 fs) longitudinal resolution and $0.01 \%$ energy resolution. The measured 2D longitudinal phase space will be used to train a virtual diagnostic for nondestructive phase space prediction and tuning [12].

\section{Differential pumping}

Because of the dramatically increased intensity and improved transverse emittance of FACET-II beams, the use of windows to isolate the plasma region at the FACET-II experimental area is not possible. A differential pumping system is designed to address this issue. It consists of an upstream and downstream section to isolate up to 5 Torr of helium buffer gas in the experimental area from the rest of the accelerator. The upstream system reaches $\sim 10^{-9}$ Torr as required for high-voltage operation of the deflecting cavity. The target vacuum for the downstream section is $10-100$ mTorr, to prevent degradation of the accelerated bunch emittance prior to measurement.

The design for the upstream section uses four stages of pumping, each utilizing a turbo-molecular pump with a peak pumping rate of $\sim 2200 \mathrm{l} / \mathrm{s}$ of helium at an input pressure below mTorr. To be compatible with beam focusing

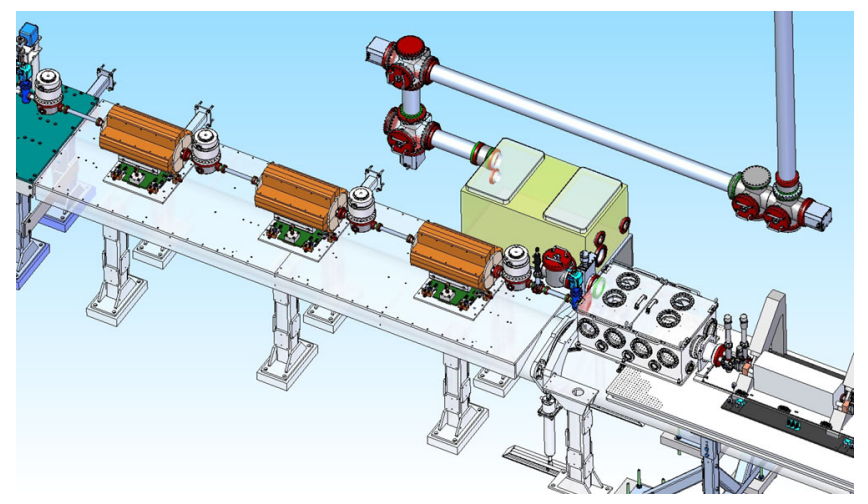

FIG. 10. Final focus triplet including a differential pumping system and experimental area laser layout. 


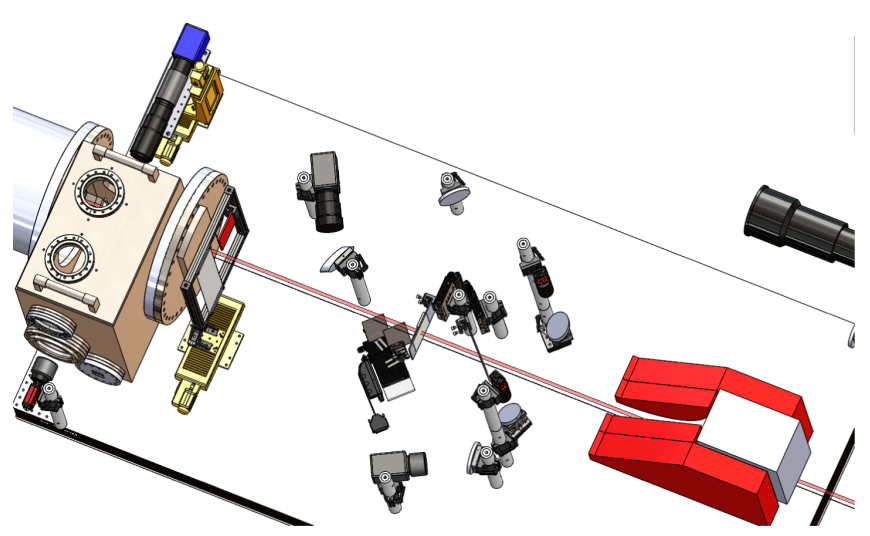

FIG. 11. The reimaging spectrometer diagnostics. From left to right: in-vacuum OTR, phosphor screen for electron and gammaradiation profiles, Cherenkov light profile monitor, a set of phosphor screens for spectral measurements of gamma radiation, and the magnetic coils for the Compton spectrometer.

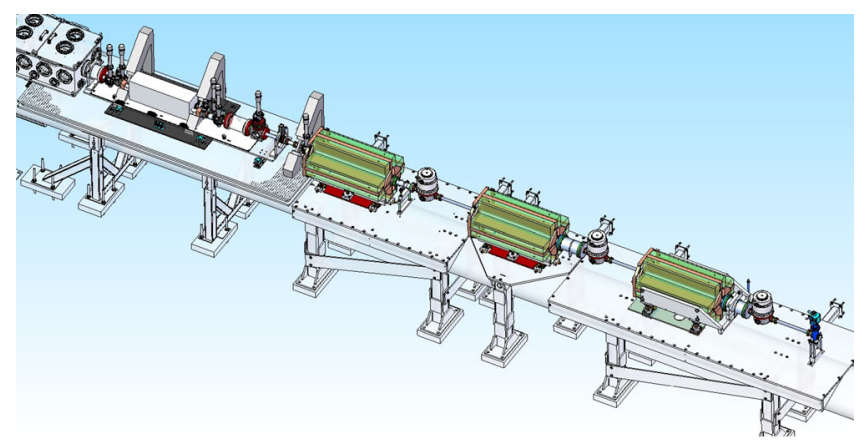

FIG. 12. Downstream differential pumping and imaging spectrometer quadrupole magnets.

optics, the three upstream sections use conductance-limiting round tubes with $18 \mathrm{~mm}$ diameter and $700 \mathrm{~mm}$ length, while the first stage, closest to the experimental area, uses a $5 \mathrm{~mm} \times 100 \mathrm{~mm}$ tube. Differential pumping apertures are designed to be remotely removable to meet the reduced background requirements of SFQED experiments. The downstream differential pumping system is identical to the first three upstream sections. Locations of the pumps are shown in Figs. 10 and 12.

\section{CONCLUSIONS}

The FACET-II facility at SLAC National Accelerator Laboratory will be a National User Facility supporting an experimental program that expands and improves upon the successful program conducted at FACET. FACET-II will upgrade the existing SLAC linac in sectors 10-20 to include a new electron rf photoinjector and two new bunch compressors in sectors 11 and 20. Starting in 2020, this will allow the resumption of research into electron beam-driven plasma acceleration, additional programs of other advanced acceleration $R \& D$, and experiments probing the fundamental properties of nature. In addition to improvements in the SLAC linac, various upgrades to the sector 20 experimental area and advanced, novel beam diagnostics systems are planned to facilitate the experimental programs. A number of enhancements including a new compact damping ring to be installed in the SLAC linac tunnel are foreseen to allow the resumption of positron experiments, starting from the 2022 timeframe.

\section{ACKNOWLEDGMENTS}

This work was supported by U.S. Department of Energy under Contract No. DE-AC02-76SF00515.

[1] I. Blumenfeld, C. E. Clayton, F.-J. Decker, M. J. Hogan, C. Huang, R. Ischebeck, R. Iverson, C. Joshi, T. Katsouleas, N. Kirby, W. Lu, K. A. Marsh, W. B. Mori, P. Muggli, E. Oz, R. H. Siemann, D. Walz, and M. Zhou, Energy doubling of $42 \mathrm{GeV}$ electrons in a metre-scale plasma wakefield accelerator, Nature (London) 445, 741 (2007).

[2] M. Litos et al., High-efficiency acceleration of an electron beam in a plasma wakefield accelerator, Nature (London) 515, 92 (2014).

[3] S. Corde et al. Multi-gigaelectronvolt acceleration of positrons in a self-loaded plasma wakefield, Nature (London) 524, 442 (2015).

[4] S. Gessner et al., Demonstration of a positron beam-driven hollow channel plasma wakefield accelerator, Nat. Commun. 7, 11785 (2016).

[5] C. E. Clayton, E. Adli, J. Allen, W. An, C. I. Clarke, S. Corde, J. Frederico, S. Gessner, S. Z. Green, M. J. Hogan, C. Joshi, M. Litos, W. Lu, K. A. Marsh, W. B. Mori, N. Vafaei-Najafabadi, X. Xu, and V. Yakimenko, Selfmapping the longitudinal field structure of a nonlinear plasma accelerator cavity, Nat. Commun. 7, 12483 (2016).

[6] R. D'Arcy et al., Tunable Plasma-Based Energy Dechirper, Phys. Rev. Lett. 122, 034801 (2019).

[7] E. Adli et al. Acceleration of electrons in the plasma wakefield of a proton bunch, Nature (London) 561, 363 (2018).

[8] Advanced Accelerator Development Strategy report, Report of the Roadmap Workshop 2016, G. Blazey (chair), http://science.energy.gov/hep/community-resources/ reports/.

[9] Linac Coherent Light Source (LCLS) Report No. SLAC-R593, 2002.

[10] C. Joshi, E. Adli, W. An, C. E. Clayton, S. Corde, S. Gessner, M. J. Hogan, M. Litos, W. Lu, K. A. Marsh, W. B. Mori, N. Vafaei-Najafabadi, B. O'shea, Xinlu Xu, G. White, and V. Yakimenko, Plasma wakefield acceleration experiments at FACET II, Plasma Phys. Controlled Fusion 60, 034001 (2018).

[11] A. Deng et al., Electron bunch generation from a plasma photocathode, Nat. Phys. 1745 (2019).

[12] C. Emma, A. Edelen, M. J. Hogan, B. O'Shea, G. White, and V. Yakimenko, Machine learning-based longitudinal phase space prediction of particle accelerators, Phys. Rev. Accel. Beams 21, 112802 (2018). 
[13] A. Doche et al., Acceleration of a trailing positron bunch in a plasma wakefield accelerator, Sci. Rep. 7, 14180 (2017).

[14] C. Lindström et al., Measurement of Transverse Wakefields Induced by a Misaligned Positron Bunch in a Hollow Channel Plasma Accelerator, Phys. Rev. Lett. 120, 124802 (2018).

[15] B. D. O'Shea, G. Andonian, S. K. Barber, K. L. Fitzmorris, S. Hakimi, J. Harrison, P. D. Hoang, M. J. Hogan, B. Naranjo, O. B. Williams, V. Yakimenko, and J. B. Rosenzweig, Observation of acceleration and deceleration in gigaelectron-volt-per-metre gradient dielectric wakefield accelerators, Nat. Commun. 7, 12763 (2016).

[16] R. Lee and M. Lampe, Electromagnetic Instabilities, Filamentation, and Focusing of Relativistic Electron Beams, Phys. Rev. Lett. 31, 1390 (1973).

[17] B. Allen, V. Yakimenko, M. Babzien, M. Fedurin, K. Kusche, and P. Muggli, Experimental Study of Current Filamentation Instability, Phys. Rev. Lett. 109, 185007 (2012).

[18] A. Benedetti, M. Tamburini, and C. H. Keitel, Giant collimated gamma-ray flashes, Nat. Photonics 12, 319 (2018).

[19] FACET-II TDR, Report No. SLAC-R-1072.

[20] G. R. White, Y. Cai, R. Hettel, M. Johansson, V. Yakimenko, and G. Yocky, A compact $335 \mathrm{MeV}$ positron damping ring design for FACET-II, in Proceedings of IPAC2017, Copenhagen, Denmark (JACoW, 2017), http:// accelconf.web.cern.ch/AccelConf/ipac2017/.

[21] S. B. van der Geer and M. J. de Loos, The General Particle Tracer code, 2001, http://www.pulsar.nl/gpt/.

[22] D. Sagan, BMAD software toolkit for charged-particle and X-ray simulations, https://www.classe.cornell.edu/bmad/.
[23] P. Tenenbaum, SLAC National Accelerator Laboratory Report No. SLAC-PUB-11215, 2005, http://www.slac .stanford.edu/accel/ilc/codes/Lucretia/.

[24] Y. K. Semertzidis, R. Burns, V. Castillo, R. Larsen, D. M. Lazarus, D. Nikas, C. Ozben, T. Srinivasan-Rao, A. Stillman, T. Tsang, and L. Kowalski, A single shot, sub-picosecond beam bunch characterization with electro-optic techniques, in Proceedings of the 18th Particle Accelerator Conference, New York, 1999 (IEEE, New York, 1999), p. 490.

[25] P. Muggli, K. A. Marsh, S. Wang, C. E. Clayton, S. Lee, T. C. Katsouleas, and C. Joshi, Photo-ionized lithium source for plasma accelerator applications, IEEE Trans. Plasma Sci. 27, 791 (1999).

[26] http://www.fastlite.com/en/.

[27] R. Weingartner, S. Raith, A. Popp, S. Chou, J. Wenz, K. Khrennikov, M. Heigoldt, A. R. Maier, N. Kajumba, M. Fuchs, B. Zeitler, F. Krausz, S. Karsch, and F. Grüner, Ultralow emittance electron beams from a laser-wakefield accelerator, Phys. Rev. Accel. Beams 15, 111302 (2012).

[28] B. O'Shea, Diffraction and detector considerations for high resolution imaging, SLAC National Accelerator Laboratory Report No. SLAC-TN-18-001.

[29] E. Adli, S. J. Gessner, S. Corde, M. J. Hogan, and H. H. Bjerke, Cherenkov light-based beam profiling for ultrarelativistic electron beams, Nucl. Instrum. Methods Phys. Res., Sect. A 783, 35 (2015).

[30] Y. Sakai et al., Single shot, double differential spectral measurements of inverse Compton scattering in the nonlinear regime, Phys. Rev. Accel. Beams 20, 060701 (2017). 